overall cancer incidence and 4 in the structure of the overall cancer mortality. Since 2008, population BC screening has been introduced among women 50-60 years old with an interval of 2 years. In 2014, 80\% of mammography devices were digitized. Since 2018, the screening age has been extended to $40-70$ years.

Methodology The following screening indicators were analyzed: the cancer detection rate (CDR), the proportion of $0-1$ stages (since 2011), the interrelation with the dynamics of morbidity and mortality. According to the levels of morbidity (average annual rate for 2004-2007), the regions of the country are divided into three groups: A - high (23.2-30.8 per 100000 population), B - mid (18.4\%ooo-22.5\%ooo), and C low $(13,2-15.18 \%$ ooo) rate.

In total, over 12 years of screening, 5,763,518 women were examined, 9,323 cases of BC were identified. Coverage to the targeted population ranged from $82 \%$ in 2012 to $47.5 \%$ in 2018. Since 2011, BC screening cases (8 330) have been compared with the cancer registry.

Results Average CDR in 2011-2019 was 0.18\%, in groups A - $0.23 \%, \mathrm{~B}-0.20 \%$ and C $-0.14 \%$. The share of 0 -I stages was $36.2 \%$, in groups A - 39.9\%, B - 29.9\% and C - 22.1\%.

The average annual baseline morbidity (2004-2007) was in groups A - 26.6 per 100000 population, B - 21.2\%ooo and C -14.3\%ooo. Before digitalization (2008-2014), the average annual morbidity in groups was: in A - 30.5\%ooo, B - 22.8\% ooo, C - 16.5\%ooo, after digitalization, respectively 31.5 , 23.8 and 18.7 .

The greatest increase of morbidity was noted in groups A and C (20.6\% and 30.7\%), less in group B (12.4\%). Screening increased the incidence since $20.8 \%$ ooo in 2008 till 25.3 in 2018 and slightly reduced the mortality rate from 8.5 (2008) to 6.8 (2018) per 100 thousand of the population.

Conclusion Over the 12 years of screening in Kazakhstan, the BC incidence has increased and mortality has decreased. Regions with a high baseline morbidity had higher CDRs by screening, especially in the first years, as well as high levels of BC detection in stage 0 -I. It is possible that radiologists have better skills and women's cancer awareness is higher in regions with a high cancer incidence.

For regions with different BC incidence rates, it is necessary to identify indicators to assess of the effectiveness and improve the quality of screening.

Disclosures The authors have no financial conflicts of interest.

\section{A RETROSPECTIVE STUDY OF STERNAL METASTASES IN BREAST CANCER}

Cherifa Fazila Ghomari, Abdelkader Medjahedi. Tlemcen University Hospital. Dr Tidjani Damerdji; Université Tlemcen, Medicine Departement; Nuclear Medicine

\subsection{6/ijgc-2020-ESG0.4}

Introduction/Background Sternal metastases of breast cancer are rare. Their occurrence is due to the spread of malignant cells via the hematogenous route or via a local pathway from the internal mammary nodes.

The aim of this study is to define the different pattern of sternal malignant abnormalities on bone scan.

Methodology It is a retrospective study including breast cancer patients, referred for bone scan during 2019, at the Nuclear Medicine department of Tlemcen University Hospital in Algeria.
Two hours after the intravenous injection of $8-10 \mathrm{MBq} / \mathrm{kg}$ 99mTc-HMDP, whole body scanning is accomplished by dual head hybrid gamma camera with low energy high resolution collimator. The SPECT/CT (single photon emission tomography/computed tomography) acquisition is used to better characterize the presence, location, and extent of disease in some patients.

Results A total of 54 malignant sternal abnormalities were found in 500 breast cancer patients (10,8\%).

Half of the lesions (27 cases) were located in the sternal body, $17(32 \%)$ in the manubrium and $10(18 \%)$ in the entire bone structure.

The majority of sternal abnormalities (47 cases; 87\%) was found as a part of multiple metastases, while only 2 cases $(4 \%)$ as a part of oligometastases and 5 cases (9\%) as the initial site of bone metastases.

A solitary sternal uptake on bone scan is difficult to interpret due to various etiologies, both benign and malignant. The SPECT/CT acquisition has allowed us to define the secondary origin of the radioactivity uptake after cross sectional study and confrontation with morphological imaging.

The predominant scintigraphic pattern was that of hot lesions (48 cases; 89\%), which highlights an osteoblastic hyperactivity. A cold lesion representing an osteoclastic activity, is rarely seen in bone scan until it is surrounded by an increased radioactivity uptake. The latter aspect was found in 6 patients $(11 \%)$.

Conclusion Radionuclide bone scintigraphy is a useful tool for recognizing sternal abnormalities in breast cancer patients.

Disclosures We have no disclosures.

\section{AXILLARY LYMPHADENECTOMY VS. SENTINEL NODE BIOPSY FOR EARLY-STAGE CLINICALLY NODE-NEGATIVE BREAST CANCER: A SYSTEMATIC REVIEW AND META- ANALYSIS}

\begin{abstract}
${ }^{1}$ Stamatios Petousis, ${ }^{2}$ Panagiotis Christidis, ${ }^{3}$ Chrysoula Margioula-Siarkou, ${ }^{3}$ Anastasios Liberis, ${ }^{3}$ Eleftherios Vavouldis, ${ }^{3}$ Georgia Margioula-Siarkou, ${ }^{3}$ Alexios Papanikolaou, ${ }^{3}$ George Mavromatidis, ${ }^{3}$ Konstantinos Dinas. ${ }^{1} 2 n d$ Department of Obstetrics and Gynaecology, Aristotle University of Thessaloniki; ${ }^{2} 4$ th Department of General Surgery, Aristotle University of Thessaloniki, Grrece; ${ }^{3} 2$ nd Department of Obstetrics and Gynaecology, Aristotle University of Thessaloniki
\end{abstract}

\subsection{6/ijgc-2020-ESGO.5}

Introduction/Background Axillary lymph node dissection had been for years the gold standard for surgical staging and locoregional control of axilla in early-stage breast cancer patients. However, sentinel node biopsy has been placed in early 1990 s as an effective alternative method of surgical staging. Main objective of the study is to compare oncological and survival outcomes between systematic axillary lymph node dissection (ALND) vs sentinel lymph node and axillary lymphadenectomy only if sentinel positive (SLN \pm ALND) in early-stage, clinically node-negative breast cancer patients.

Methodology A systematic review and meta-analysis adhered to PRISMA guidelines was performed. Included studies were prospective randomized controlled trials (RCTs) comparing survival outcomes of ALND vs. SLN \pm ALND in early-stage, node-negative breast cancer patients. Patients enrolled were only those with tumor size lower than $4 \mathrm{~cm}$, clinically negative nodes and treated with breast-conservative surgery. Primary outcomes were locoregional recurrence, overall death and cancer-related death. 